\title{
P04-1-135
}

Poster session

\section{Investigation of the effect of Agmatine in Rotenone induced Parkinson's Disease Model in Rats}

\section{Caner Gunaydin ${ }^{1}$, Mehmet Emin Onger ${ }^{2}$, Bahattin Avci ${ }^{3}$, Ayhan Bozkurt $^{4}$, Nihan Cankara ${ }^{5}$, Suleyman Sirri Bilge ${ }^{1}$}

${ }^{1}$ Pharmacology, Ondokuz Mayis University School of Medicine, Turkey, ${ }^{2}$ Histology and Embryology, Ondokuz Mayis University School of Medicine, Turkey, ${ }^{3}$ Biochemistry, Ondokuz Mayis University School of Medicine, Turkey, ${ }^{4}$ Physiology, Ondokuz Mayis University School of Medicine, Turkey, ${ }^{5}$ Pharmacology, Suleyman Demirel University School of Medicine, Turkey

Introduction: Agmatine is an endogenous decarboxylation product which is obtained from arginine by argininedecarboxylase. Exogenously administered agmatine was demonstrated as neuroprotective, anti-oxidant and antidepressant in experimental animal models. The aim of the current study was to investigate the effect of agmatine in rotenone induced Parkinson model in rats.

Method: Male Sprague-Dawley rats $(250-280 \mathrm{~g})$ were used. Agmatine $(100 \mathrm{mcg} / 5 \mathrm{mcl}$, i.c.v. $)$ was administered daily for 17 days. Seven days after the first agmatine administration, rotenone was administreted $(0.5 \mathrm{mcg} / 1 \mathrm{mcl})$ to create Parkinson's disease model. Rats were rotated with apomorphine 10 days after rotenone administration for successful validation of Parkinson's disease model. Motor coordination of rats were evaluated with rotarod and pole test one day after the validation of Parkinson's disease, just before the decapitation. Brain tissues were taken for the biochemical and histopathological evaluations. The data were analysed by one-way analysis of variance (ANOVA) followed by Bonferroni and Tukey's multiple comparison test. Statistical significance was considered at $\mathrm{P}<0.05$ in all the cases.

Results: Agmatine administration significantly reduced apomorphine induced turning behaviour $(\mathrm{p}<0.05)$. Also, pole test duration was significantly shorten in agmatine administered rats $(\mathrm{p}<0.05)$. Agmatine administration increased catalase and superoxide dismutase levels, and it attenuated rotenone induced MDA increase and BDNF decrease $(\mathrm{p}<0.05-0.01)$. Rotenone induced increases in histopathological damage scores were alleviated in agmatine administered rats $(p<0.05$ $-0.01)$.

Conclusion: These results suggest that agmatine has neuroprotective and anti-oxidant effects in rotenone-induced Parkinson's disease model.

Keywords : Agmatine, Parkinson's disease, Rotenone, Rat 